\title{
Exact Solution of Navier-Stokes Equations
}

\author{
Sangwha-Yi
}

Department of Math, Taejon University, South Korea

*Corresponding Author: Sangwha-Yi, Department of Math, Taejon University, South Korea

Abstract: In Navier-Stokes equations (NASA's Navier-Stokes Equations, 3-dimensional-unsteady), we discover the exact solution by Newton potential function and time-function. We think the solution likely Newton potential function that be able to solve Laplace equation.

Keywords: Navier-Stokes Equations; Exact solutions; Newton potential function; Time function

PACS Number: $04,04.90 .+e, 47 \cdot 10 .-g$

\section{INTRODUCTION}

We discover the exact solution in Navier-Stokes equation by Newton potential function and time function.

According NASA's Navier-Stokes Equations (3-dimensional-unsteady),

Coordinate: $(x, y, z)$, Time: $t$, Pressure: $p$, Heat Flux: $q$

Density: $\rho$, Stress: $\tau$, Reynolds Number: $R_{e}$,

Velocity Components: $(u, V, w)$ Total Energy: $E_{t}$ Plandtl Number: $P_{r}$

Continuity: $\frac{\partial \rho}{\partial t}+\frac{\partial(\rho u)}{\partial x}+\frac{\partial(\rho v)}{\partial y}+\frac{\partial(\rho w)}{\partial z}=0$

X-Momentum: $\frac{\partial(\rho u)}{\partial t}+\frac{\partial\left(\rho u^{2}\right)}{\partial x}+\frac{\partial(\rho u v)}{\partial y}+\frac{\partial(\rho u w)}{\partial z}$

$$
=-\frac{\partial p}{\partial x}+\frac{1}{R_{e}}\left[\frac{\partial \tau_{x x}}{\partial x}+\frac{\partial \tau_{x y}}{\partial y}+\frac{\partial \tau_{x z}}{\partial z}\right]
$$

Y-Momentum: $\frac{\partial(\rho v)}{\partial t}+\frac{\partial(\rho u v)}{\partial x}+\frac{\partial\left(\rho v^{2}\right)}{\partial y}+\frac{\partial(\rho v W)}{\partial z}$

$$
=-\frac{\partial p}{\partial y}+\frac{1}{R_{e}}\left[\frac{\partial \tau_{x y}}{\partial x}+\frac{\partial \tau_{y y}}{\partial y}+\frac{\partial \tau_{y z}}{\partial z}\right]
$$

Z-Momentum: $\frac{\partial(\rho W)}{\partial t}+\frac{\partial(\rho u w)}{\partial x}+\frac{\partial(\rho W v)}{\partial y}+\frac{\partial\left(\rho w^{2}\right)}{\partial z}$

$$
=-\frac{\partial p}{\partial z}+\frac{1}{R_{e}}\left[\frac{\partial \tau_{x z}}{\partial x}+\frac{\partial \tau_{y z}}{\partial y}+\frac{\partial \tau_{z z}}{\partial z}\right]
$$


Energy: $\frac{\partial E_{t}}{\partial t}+\frac{\partial\left(E_{t} u\right)}{\partial x}+\frac{\partial\left(E_{t} v\right)}{\partial y}+\frac{\partial\left(E_{t} w\right)}{\partial z}=-\frac{\partial(p u)}{\partial x}-\frac{\partial(p v)}{\partial y}-\frac{\partial(p w)}{\partial z}$

$$
\begin{aligned}
&- \frac{1}{R_{e} P_{r}}\left[\frac{\partial q_{x}}{\partial x}+\frac{\partial q_{y}}{\partial y}+\frac{\partial q_{z}}{\partial z}\right] \\
&+\frac{1}{R_{e}}\left[\frac{\partial}{\partial x}\left(u \tau_{x x}+v \tau_{x y}+w \tau_{x z}\right)+\frac{\partial}{\partial y}\left(u \tau_{x y}+v \tau_{y y}+w \tau_{y z}\right)+\frac{\partial}{\partial z}\left(u \tau_{x z}+v \tau_{y z}+w \tau_{z z}\right)\right]
\end{aligned}
$$

\section{EXACT SOLUTION IN 3-DIMENTIONAL NAVIER-STOKES EQUATION (INCLUDE TIME)}

For we solve equations, we use Newton potential function and time function. If we think the solution of Laplace equation,

$$
\begin{aligned}
u=\frac{C_{1}}{r^{3}} \times f(t), v & =\frac{C_{1}}{r^{3}} y f(t), w=\frac{C_{1}}{r^{3}} z f(t), r=\sqrt{x^{2}+y^{2}+z^{2}} \\
\rho & =\rho_{0}, r>0
\end{aligned}
$$

In this case, we solve $\mathrm{Eq}(1)$.

$$
\frac{\partial \rho}{\partial t}+\frac{\partial(\rho u)}{\partial x}+\frac{\partial(\rho v)}{\partial y}+\frac{\partial(\rho w)}{\partial z}=f(t) \rho_{0}\left[-\frac{3 C_{1}}{r^{5}}\left(x^{2}+y^{2}+z^{2}\right)+\frac{3 C_{1}}{r^{3}}\right]=0
$$

Second point, in $\mathrm{Eq}(2)$

$$
\begin{aligned}
& \tau_{x x}=\frac{C_{2}}{r^{6}} x^{2}[f(t)]^{p}, \tau_{x y}=\frac{C_{2}}{r^{6}} x y[f(t)]^{f}, \quad \tau_{x z}=\frac{C_{2}}{r^{6}} x z[f(t)]^{p}, \\
& , \ldots, \tau_{i j}=\frac{C_{2}}{r^{6}} x^{i} x^{j}[f(t)]^{f}, r>0 \\
& \frac{\partial(\rho u)}{\partial t}+\frac{\partial\left(\rho u^{2}\right)}{\partial x}+\frac{\partial(\rho u v)}{\partial y}+\frac{\partial(\rho u w)}{\partial z} \\
& =\rho_{0} \dot{f}(t) \frac{C_{1}}{r^{3}} x+[f(t)]^{2} \rho_{0}\left[-\frac{6 C_{1}^{2}}{r^{8}}\left(x^{3}+x y^{2}+x z^{2}\right)+\frac{C_{1}^{2}}{r^{6}}(2 x+x+x)\right] \\
& =\rho_{0} \dot{f}(t) \frac{C_{1}}{r^{3}} x-[f(t)]^{R} \rho_{0} \frac{2 C_{1}^{2}}{r^{6}} x
\end{aligned}
$$

In this time, if $f(t)$ is,

$$
\frac{1}{[f(t)]^{2}} \frac{d}{d t}[f(t)]=1 \rightarrow f(t)=\frac{1}{C^{1}-t}, t \geq 0, C^{\prime}<0 \text { is constant }
$$

Therefore,

$$
\begin{aligned}
& =\rho_{0} \frac{1}{\left(C^{\prime}-t\right)^{2}} \frac{C_{1}}{r^{3}} x-\frac{1}{\left(C^{\prime}-t\right)^{2}} \rho_{0} \frac{2 C_{1}^{2}}{r^{6}} x \\
& =-\frac{\partial p}{\partial x}+\frac{1}{R_{e}}\left[\frac{\partial \tau_{x x}}{\partial x}+\frac{\partial \tau_{x y}}{\partial y}+\frac{\partial \tau_{x z}}{\partial z}\right]
\end{aligned}
$$




$$
\begin{aligned}
& =-\frac{\partial p}{\partial x}+\frac{1}{R_{e}}\left[-\frac{6 C_{2}}{r^{8}}\left(x^{3}+x y^{2}+x z^{2}\right)+\frac{C_{2}}{r^{6}}(2 x+x+x)\right] \frac{1}{\left(C^{1}-t\right)^{2}} \\
& =-\frac{\partial p}{\partial x}-\frac{1}{R_{e}} \frac{2 C_{2}}{r^{6}} x \frac{1}{\left(C^{1}-t\right)^{2}}
\end{aligned}
$$

Hence, in $\mathrm{Eq}(2)$,in $\mathrm{Eq}(3)$ and in $\mathrm{Eq}(4)$,

$$
\begin{aligned}
& \frac{\partial p}{\partial x}=\left[\frac{2 x}{r^{6}}\left(\rho_{0} C_{1}^{2}-\frac{C_{2}}{R_{e}}\right)-\rho_{0} \frac{C_{1}}{r^{3}} x\right] \frac{1}{\left(C^{\prime}-t\right)^{2}}=\left[\frac{2 C_{3}}{r^{6}} x-\rho_{0} \frac{C_{1}}{r^{3}} x\right] \frac{1}{\left(C^{\prime}-t\right)^{2}}, \\
& \frac{\partial p}{\partial y}=\left[\frac{2 y}{r^{6}}\left(\rho_{0} C_{1}^{2}-\frac{C_{2}}{R_{e}}\right)-\rho_{0} \frac{C_{1}}{r^{3}} y\right] \frac{1}{\left(C^{\prime}-t\right)^{2}}=\left[\frac{2 C_{3}}{r^{6}} y-\rho_{0} \frac{C_{1}}{r^{3}} y\right] \frac{1}{\left(C^{\prime}-t\right)^{2}}, \\
& \frac{\partial p}{\partial z}=\left[\frac{2 z}{r^{6}}\left(\rho_{0} C_{1}^{2}-\frac{C_{2}}{R_{e}}\right)-\rho_{0} \frac{C_{1}}{r^{3}} z\right] \frac{1}{\left(C^{\prime}-t\right)^{2}}=\left[\frac{2 C_{3}}{r^{6}} z-\rho_{0} \frac{C_{1}}{r^{3}} z\right] \frac{1}{\left(C^{\prime}-t\right)^{2}} C_{3}=\rho_{0} C_{1}^{2}-\frac{C_{2}}{R_{e}}
\end{aligned}
$$

Therefore,

$p=\left[-\frac{1}{2} \frac{C_{3}}{r^{4}}+\rho_{0} \frac{C_{1}}{r}\right] \frac{1}{\left(C^{1}-t\right)^{2}}, C_{3}=\rho_{0} C_{1}^{2}-\frac{C_{2}}{R_{e}}, r>0$

In $\operatorname{Eq}(5)$, if $E_{t}, q_{i}$ is,

$$
\begin{aligned}
& E_{t}=E_{0}, q_{x}=\frac{C_{4}}{r^{4}} \frac{x}{\left(C^{1}-t\right)^{3}}, q_{y}=\frac{C_{4}}{r^{4}} y \frac{1}{\left(C^{1}-t\right)^{3}}, q_{z}=\frac{C_{4}}{r^{4}} z \frac{1}{\left(C^{1}-t\right)^{3}}, r>0 \\
& \frac{\partial E_{t}}{\partial t}+\frac{\partial\left(E_{t} u\right)}{\partial x}+\frac{\partial\left(E_{t} v\right)}{\partial y}+\frac{\partial\left(E_{t} W\right)}{\partial z}=0
\end{aligned}
$$

As,

$$
(p u, p v, p w)=\left[-\frac{1}{2} \frac{C_{1} C_{3}}{r^{7}}+\rho_{0} \frac{C_{1}^{2}}{r^{4}}\right](x, y, z) \frac{1}{\left(C^{1}-t\right)^{3}}, r>0
$$

Hence,

$$
\begin{aligned}
& -\frac{\partial(p u)}{\partial x}-\frac{\partial(p v)}{\partial y}-\frac{\partial(p w)}{\partial z} \\
& =\left[\left\{-7 \frac{C_{3} C_{1}}{2 r^{9}}\left(x^{2}+y^{2}+z^{2}\right)+3 \frac{C_{3} C_{1}}{2 r^{7}}\right\}+\left\{4 \rho_{0} \frac{C_{1}^{2}}{r^{6}}\left(x^{2}+y^{2}+z^{2}\right)-3 \rho_{0} \frac{C_{1}^{2}}{r^{4}}\right\}\right] \frac{1}{\left(C^{1}-t\right)^{3}} \\
& =\left[-\frac{2 C_{1} C_{3}}{r^{7}}+\rho_{0} \frac{C_{1}^{2}}{r^{4}}\right] \frac{1}{\left(C^{\prime}-t\right)^{3}}
\end{aligned}
$$

So,

$$
\begin{gathered}
-\frac{1}{R_{e} P_{r}}\left[\frac{\partial q_{x}}{\partial x}+\frac{\partial q_{y}}{\partial y}+\frac{\partial q_{z}}{\partial z}\right] \\
=-\frac{1}{R_{e} P_{r}}\left[\left\{-\frac{4 C_{4}}{r^{6}}\left(x^{2}+y^{2}+z^{2}\right)+\frac{3 C_{4}}{r^{4}}\right\} \frac{1}{\left(C^{\prime}-t\right)^{3}}\right]=\frac{1}{R_{e} P_{r}} \frac{C_{4}}{r^{4}} \frac{1}{\left(C^{\prime}-t\right)^{3}}
\end{gathered}
$$

In this time, 


$$
\begin{gathered}
\tau_{i j}=\frac{C_{2}}{r^{6}} x^{i} x^{j}[f(t)]^{p}=\frac{C_{2}}{r^{6}} x^{i} x^{j} \frac{1}{\left(C^{1}-t\right)^{2}}, r>0 \\
\left(\tau_{x j} u, \tau_{y j} v, \tau_{z} w\right)=\frac{C_{1} C_{2}}{r^{9}}\left(x^{2}, y^{2}, z^{2}\right) x^{j} \frac{1}{\left(C^{1}-t\right)^{3}}, r>0, t \geq 0, C^{\prime}<0 \text { is constant } \\
\frac{1}{R_{e}}\left[\frac{\partial}{\partial x}\left(u \tau_{x x}+v \tau_{x y}+w \tau_{x z}\right)+\frac{\partial}{\partial y}\left(u \tau_{x y}+v \tau_{y y}+w \tau_{y z}\right)+\frac{\partial}{\partial z}\left(u \tau_{x z}+v \tau_{y z}+w \tau_{z z}\right)\right] \\
=\frac{1}{R_{e}}\left[-9 \frac{C_{1} C_{2}}{r^{11}} x^{2}\left(x^{2}+y^{2}+z^{2}\right)+\frac{C_{1} C_{2}}{r^{9}}\left(3 x^{2}+y^{2}+z^{2}\right)\right. \\
-9 \frac{C_{1} C_{2}}{r^{11}} y^{2}\left(x^{2}+y^{2}+z^{2}\right)+\frac{C_{1} C_{2}}{r^{9}}\left(x^{2}+3 y^{2}+z^{2}\right) \\
\left.-9 \frac{C_{1} C_{2}}{r^{11}} z^{2}\left(x^{2}+y^{2}+z^{2}\right)+\frac{C_{1} C_{2}}{r^{9}}\left(x^{2}+y^{2}+3 z^{2}\right)\right] \frac{1}{\left(C^{\prime}-t\right)^{3}} \\
=\frac{1}{R_{e}}\left[-9 \frac{C_{1} C_{2}}{r^{7}}+5 \frac{C_{1} C_{2}}{r^{7}}\right] \frac{1}{\left(C^{\prime}-t\right)^{3}}=-\frac{1}{R_{e}} \frac{4 C_{1} C_{2}}{r^{7}} \frac{1}{\left(C^{\prime}-t\right)^{3}}
\end{gathered}
$$

Hence, $\mathrm{Eq}(5)$ is

$$
\begin{aligned}
& 0=\left[\left(-2 \frac{C_{1} C_{3}}{r^{7}}+\rho_{0} \frac{C_{1}^{2}}{r^{4}}\right)+\frac{1}{R_{e} P_{r}} \frac{C_{4}}{r^{4}}-\frac{1}{R_{e}} \frac{4 C_{1} C_{2}}{r^{7}}\right] \frac{1}{\left(C^{1}-t\right)^{3}}, \\
& C_{3}=-\frac{2 C_{2}}{R_{e}} \quad C_{4}=-\rho_{0} C_{1}^{2} R_{e} P_{r} \\
& C_{3}=\rho_{0} C_{1}^{2}-\frac{C_{2}}{R_{e}}=-\frac{2 C_{2}}{R_{e}}, C_{2}=-\rho_{0} R_{e} C_{1}^{2}
\end{aligned}
$$

Therefore, $P$ is

$$
\begin{aligned}
& p=\left[-\frac{1}{2} \frac{C_{3}}{r^{4}}+\rho_{0} \frac{C_{1}}{r}\right] \frac{1}{\left(C^{\prime}-t\right)^{2}}=\left[\frac{C_{2}}{R_{e}} \frac{1}{r^{4}}+\rho_{0} \frac{C_{1}}{r}\right] \frac{1}{\left(C^{\prime}-t\right)^{2}}=\left[-\rho_{0} \frac{C_{1}^{2}}{r^{4}}+\rho_{0} \frac{C_{1}}{r}\right] \frac{1}{\left(C^{\prime}-t\right)^{2}} \\
& C_{4}=-\rho_{0} C_{1}^{2} R_{e} \rho_{r}, q_{x}=\frac{C_{4}}{r^{4}} \times \frac{1}{\left(C^{\prime}-t\right)^{3}}=-\rho_{0} \frac{C_{1}^{2} R_{e} \rho_{r}}{r^{4}} \frac{x}{\left(C^{\prime}-t\right)^{3}} \\
& a_{y}=\frac{C_{4}}{r^{4}} y \frac{1}{\left(C^{\prime}-t\right)^{3}}=-\rho_{0} \frac{C_{1}^{2} R_{e} \rho_{r}}{r^{4}} y \frac{1}{\left(C^{\prime}-t\right)^{3}} q_{z}=\frac{C_{4}}{r^{4}} z \frac{1}{\left(C^{\prime}-t\right)^{3}}=-\rho_{0} \frac{C_{1}^{2} R_{e} \rho_{r}}{r^{4}} z \frac{1}{\left(C^{\prime}-t\right)^{3}} \\
& r>0, C^{\prime}<0, t \geq 0
\end{aligned}
$$

\section{CONCLUSION}

Therefore, the exact solution of Navier-Stokes equations (3-dimensional-unsteady) is

Pressure: $p=\left[-\rho_{0} \frac{C_{1}^{2}}{r^{4}}+\rho_{0} \frac{C_{1}}{r}\right] \frac{1}{\left(C^{\prime}-t\right)^{2}}, r>0, C^{\prime}<0, t \geq 0$

Heat Flux: 


$$
\begin{aligned}
& q_{x}=-\rho_{0} \frac{C_{1}^{2} R_{e} P_{r}}{r^{4}} \frac{x}{\left(C^{\prime}-t\right)^{3}}, q_{y}=-\rho_{0} \frac{C_{1}^{2} R_{e} P_{r}}{r^{4}} \frac{y}{\left(C^{1}-t\right)^{3}}, q_{z}=-\rho_{0} \frac{C_{1}^{2} R_{e} P_{r}}{r^{4}} \frac{z}{\left(C^{\prime}-t\right)^{3}} \\
& r>0, C^{\prime}<0, t \geq 0
\end{aligned}
$$

Density: $\rho=\rho_{0}$,

Stress: $\tau_{i j}=\frac{C_{2}}{r^{6}} x^{i} x^{j} \frac{1}{\left(C^{\prime}-t\right)^{2}}=-\rho_{0} \frac{R_{e}}{r^{6}} C_{1}^{2} x^{i} x^{j} \frac{1}{\left(C^{\prime}-t\right)^{2}}, r>0, C^{\prime}<0, t \geq 0$

Reynolds Number: $R_{e}$, Plandtl Number: $P_{r}$

Velocity Components: $(u, V, w)=\frac{C_{1}}{r^{3}}(x, y, z) \frac{1}{\left(C^{\prime}-t\right)}, r>0, C^{\prime}<0, t \geq 0$

Total Energy: $E_{t}=E_{0}$

\section{REFERENCES}

[1] Three-dimensional unsteady form of the Navier-Stokes equations: Glenn Research Center, NASA

[2] D.Yi, Ya. Sinaj, Blow ups of complex solutions of the 3-d-Navier-Stokes system and renormalization group method, J. Eur. Math. Soc 10 (2008), no. 2. 267-313

[3] I.Gallagher, M. Paicu, Remarks on the blow-up of solutions to a toy model for the Navier-Stokes equations, Proc. Amer. Math. Soc. 17 (2009), no. 6, 2075-3083

[4] N. H.Katz, A. Tapai, A note on the slightly supercritical Navier-Stokes equations in the plane, preprint

[5] S. Albeverio, A.B. Cruzeiro, Global flow and invariant (Gibbs) measures for Euler and Navier-Stokes two dimensional fluid. Commun. Math. Phys.129, 431-444(1990)

[6] A. Bensoussan, R. Temman, Equations stochastiques du type Navier-Stokes. J. Funct. Anal. 13, 195222(1973)

Citation: Sangwha-Yi. "Exact Solution of Navier-Stokes Equations", International Journal of Advanced Research in Physical Science (IJARPS), vol. 6, no. 3, pp. 39-43, 2019.

Copyright: (c) 2019 Authors, This is an open-access article distributed under the terms of the Creative Commons Attribution License, which permits unrestricted use, distribution, and reproduction in any medium, provided the original author and source are credited. 